\title{
Les frontières de l'évasion : Une taxonomie du jeu d'évasion
}

The Boundaries of Escape: A Taxonomy of Escape Game

\section{Adrien Barton et Quentin Rano}

\section{OpenEdition}

Journals

Édition électronique

URL : https://journals.openedition.org/sdj/3453

DOI : $10.4000 /$ sdj.3453

ISSN : 2269-2657

Éditeur

Laboratoire EXPERICE - Centre de Recherche Interuniversitaire Expérience Ressources Culturelles Education

\section{Référence électronique}

Adrien Barton et Quentin Rano, «Les frontières de l'évasion: Une taxonomie du jeu d'évasion », Sciences du jeu [En ligne], 16 | 2021, mis en ligne le 04 novembre 2021, consulté le 26 novembre 2021. URL : http://journals.openedition.org/sdj/3453 ; DOI : https://doi.org/10.4000/sdj.3453

Ce document a été généré automatiquement le 26 novembre 2021.

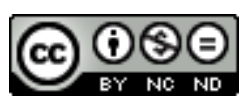

La revue Sciences du jeu est mise à disposition selon les termes de la Licence Creative Commons Attribution - Pas d'Utilisation Commerciale - Pas de Modification 4.0 International. 


\title{
Les frontières de l'évasion : Une taxonomie du jeu d'évasion
}

The Boundaries of Escape: A Taxonomy of Escape Game

\author{
Adrien Barton et Quentin Rano
}

1 Qu'est-ce qu'un jeu d'évasion (JE) ? ${ }^{1}$ Le blogue de référence sur les jeux d'évasion Room Escape Artist définit une " escape room » comme " un jeu où un groupe de participants découvre et résout en collaboration des énigmes, des tâches et des défis qui ne nécessitent aucune connaissance extérieure dans un lieu physique afin d'atteindre un objectif en un temps limité » (Spira, 2019). On pourrait être surpris que cette définition ne fasse pas intervenir la notion d'évasion. D'autres définitions précisent d'ailleurs que l'objectif est « généralement » de s'échapper d'une ou plusieurs salles (Nicholson 2015 ; Guigon et al. 2017), sans en faire une condition systématique. Mais de fait, de nombreux JE contemporains impliquent désormais des objectifs qui ne relèvent pas de l'évasion. Ainsi, l'objectif peut être par exemple de retrouver un objet précieux (comme dans Cannibal Island, Quest Factory, Paris), mettre fin à une menace (tel que dans Le Testament de l'Antiquaire, Trip Trap, Genève) ou encore libérer une tierce personne (on pensera par exemple à Très Cher Lock, Lock Academy, Paris et Toulouse).

2 Mais on pourrait aller plus loin et s'interroger si la notion de " jeu d'évasion » n'est pas plus générale que celle d' " escape room », que l'on pourrait traduire par " jeu d'évasion grandeur nature » (abrégé «JEGN»), suivant Guigon et al. (2017). Et de fait, il existe des formes de jeux apparentées qui se pratiquent de manières très différentes. On trouve entre autres les livres-jeux d'évasion (collection Escape Game, éditions Mango; collection Escape Book, 404-Editions; etc.) et les jeux d'évasion en boîte (tels que la collection Unlock!, Space Cowboys), dans lesquels les joueurs doivent également résoudre une série d'énigmes pour accomplir un objectif, et où les mécanismes typiques $\mathrm{du}$ JEGN sont reproduits de manière indirecte, par exemple via l'utilisation de cartes. Par ailleurs, on mentionne parfois le jeu vidéo en pointer-cliquer (" point-and-click» en anglais) Crimson Room (Toshimitsu Takagi, 2004), dans lequel un personnage anonyme doit parvenir à sortir d'une chambre rouge où il est enfermé, comme une influence importante des «escape rooms» contemporaines (Bucaille \& Levenq, 2018). 
D'autres jeux vidéo, comme The Room (Dodd \& Hamilton, Fireproof Games, 2012), se sont par la suite inspirés des codes de ce jeu. On pourrait vouloir regrouper certains de ces jeux (ou tous) dans la famille des «jeux d'évasion», dont le JEGN n'est qu'un des sous-types. Par ailleurs, des expériences immersives grandeur nature reprennent certains éléments du JE classique, tout en s'en éloignant par d'autres ${ }^{2}$. On pensera ainsi aux "action games» tels que Prison Island (multiples localisations), dans lequel une équipe doit résoudre une variété de tâches (notamment d'adresse et d'agilité), et reçoit un score en fin de jeu évaluant sa performance ; ou encore à un jeu comme L'Affaire du Manoir Deveaux (Panik Room, Paris), qui propose aux joueurs d'explorer un par un et les yeux bandés un manoir hanté, guidé à distance par leurs compagnons de jeux, afin de résoudre un mystère surnaturel.

3 Le JE partage certains points communs avec d'autres formes de jeux, qui ne sont pourtant pas communément qualifiées de " jeu d'évasion ». On pensera notamment aux jeux de réflexion comme des sudokus ou labyrinthes, que l'on retrouve d'ailleurs parfois dans certains JE; à certains jeux de société collaboratifs (Pandémie, Leacock, Filosofia, 2008; Sherlock Holmes - Détective Conseil, Grady, Goldberg \& Edwards, Sleuth Publications, 1981 ; etc.), dans lesquels une équipe de joueurs doit résoudre un objectif commun; au jeu de rôle (sur table ou grandeur nature), dont les soirées enquêtes ("murder party» en anglais), dans lesquelles chaque joueur incarne un personnage et doit faire la lumière sur un mystère ; aux jeux de piste et géocaching; aux chasses au trésor à distance («armchair treasure hunt» en anglais), qui ne nécessitent de se déplacer sur le terrain que pour déterrer le trésor (comme Sur la Trace de la Chouette d'Or, Valentin, Manya, 1993) ; aux jeux vidéo d'aventure en pointer-cliquer antérieurs à Crimson Room comme The Secret of Monkey Island (Gilbert, Grossman \& Schafer, Lucasfilm Games, 1990); aux jeux vidéo à énigmes (« puzzle game»), comme Myst (Miller \& Miller, Cyan, Inc., 1993), Portal (Swift, Valve, 2007) ou The Talos Principle (Hunski, Ladavac \& Tomičić, Croteam, 2014); ou encore aux « livres dont vous êtes le héros », dans lesquels le lecteur doit naviguer dans un arbre décisionnel de paragraphe en paragraphe (Solinksi, 2017). Nous aborderons donc dans cet article la question suivante : existe-t-il une nature commune aux jeux mentionnés dans le paragraphe précédent, qui justifierait de les appeler "jeux d'évasion »? Et en quoi se différencient-ils de leurs cousins plus ou moins lointains mentionnés dans ce paragraphe?

4 Toute investigation scientifique ultérieure du domaine des JE se devra d'utiliser un vocabulaire bien défini. C'est pourquoi nous proposerons ici une taxonomie du JE, à savoir une identification des termes centraux relatifs au JE, dont nous proposerons des définitions; et une classification de ces termes par des relations de généralité. Cette taxonomie reposera sur des bases méthodologiques issues du domaine de l'ontologie appliquée, que nous exposerons ci-dessous. Une analyse du champ du JE et de ses exemples prototypiques permettra de proposer une définition amendée du terme «jeu d'évasion» et de définir certains de ses sous-types ainsi que la notion centrale de « défi ».

5 Nous présenterons la méthodologie utilisée dans une deuxième partie, puis quelques classifications et ontologies de domaines connexes dans une troisième partie. Ensuite, nous explorerons la définition du JE dans une quatrième partie et celles de ses soustypes dans une cinquième partie, avant d'explorer d'autres entités centrales du JE (tâche et défi) dans une sixième partie. 


\section{Méthodologie}

6 Notre approche empruntera les méthodes de l'ontologie appliquée (Arp, Smith \& Spear, 2015 ; Masolo et al., 2003), qui définit les catégories (parfois appelées "classes", «types", " universaux» ou "concepts ») pertinentes à un domaine du savoir, et propose des axiomes formels clarifiant les relations entre ces catégories. Il existe de la sorte des ontologies appliquées dans des domaines aussi variés que la médecine, la biologie, l'ingénierie ou la géographie, ainsi que leurs sous-domaines. Certaines ontologies appliquées peuvent être représentées sous forme de fichier informatique et utilisées pour l'annotation de données, permettant de préciser de manière rigoureuse et systématique la signification de celles-ci. Plus généralement, une ontologie appliquée permet de clarifier conceptuellement les entités d'un domaine.

7 Nous ne chercherons pas ici à énoncer des axiomes formels pour les catégories pertinentes du jeu d'évasion. En revanche, nous organiserons les termes-clés du jeu d'évasion selon une structure taxonomique en nous inspirant des catégories de certaines ontologies appliquées, et utiliserons une méthode systématique d'écriture de définitions issue de l'ontologie appliquée.

Les entités représentées par une ontologie appliquée appartiennent à deux familles nettement distinctes. La première est celle de "particulier", appelé également «individu », à savoir une entité bien délimitée dans l'espace et le temps (comme cet échiquier qui se trouve sur la table basse de mon salon). La seconde est celle de "classe ", qui rassemble tous les particuliers d'un même type (comme la classe des échiquiers). On dira que l'échiquier posé sur la table de mon salon est une "instance " de la classe des échiquiers. Une taxonomie vise à représenter un ensemble de classes pertinentes du domaine, organisées par des relations de sous-classe à classe parente (dites de "subsomption»). Plus précisément, on considère généralement que $B$ est une sous-classe (ou "classe fille») de A si et seulement si toute instance de B est une instance de A. Remarquons qu'il peut $\mathrm{y}$ avoir des instances communes à deux classes sœurs (autrement dit, deux sous-classes d'une même classe) d'une taxonomie. Par exemple, dans une taxonomie où la classe « Objet matériel » aurait les deux sous-classes " Objet rouge » et "Voiture », ces deux dernières classes ont des instances communes (car il existe des voitures rouges). On remarquera au passage que les sous-classes d'une même classe peuvent différencier les instances de cette classe selon des critères différents. Par ailleurs, les sous-classes d'une taxonomie n'ont pas vocation à être exhaustives.

9 Ainsi, une taxonomie du jeu d'évasion visera à identifier les classes pertinentes dans le domaine du JE, comme «JE » et ses sous-classes, ou « défi » et ses sous-classes. Nous utiliserons, dans la mesure $\mathrm{du}$ possible, des termes proches de ceux utilisés communément. Le but n'est cependant pas d'établir une correspondance totale avec les usages linguistiques courants : toute définition systématique va s'éloigner, au moins légèrement, de certains usages habituels du langage. Notamment, certains termes (par exemple " tâche ", " défi ", « épreuve ») peuvent avoir certaines connotations dont nos définitions ne prétendent pas rendre compte.

10 Nous cherchons une définition large du JE qui englobe, comme expliqué ci-dessus, non seulement le JEGN, mais également le JE vidéo (abrégé «JEV »), le livre-JE ou le JE en boîte. Nous accepterons comme instances prototypiques de «jeu d'évasion » ce qui est reconnu comme tel par la communauté du domaine, composée notamment des acteurs 
de l'industrie du JE et des experts du sujet (blogues, réseaux sociaux tels que «The Escapers $»^{3}$, classements des meilleurs JE tels que le « Top Escape Room Project $»^{4}$, etc.). Nous ne chercherons néanmoins pas une définition tellement large qu'elle engloberait toutes les formes de jeu qui revendiquent le label de «jeu d'évasion » ou "escape game ». Comme beaucoup de termes dans l'air du temps, ceux-ci sont parfois utilisés de manière très laxiste, pour diverses raisons, notamment liées au marketing (il peut être plus vendeur de présenter un jeu comme "escape game ", même s'il n'entretient qu'un rapport très lointain avec les JE). Il faudra donc un consensus minimal de la communauté du JE pour qu'un exemple de jeu puisse être considéré comme une instance prototypique de JE.

11 Nous chercherons ainsi une définition remplissant deux critères : elle doit $\mathrm{C} 1$ ) être précise et rigoureuse, et $\mathrm{C2}$ ) inclure les exemples prototypiques de JE. On pourrait vouloir ajouter un troisième critère, à savoir qu'elle doit $\mathrm{C} 3$ ) exclure les jeux qui ne sont pas communément appelés "jeux d'évasion» dans la communauté du domaine. Toutefois, il n'est pas forcément possible de trouver une définition satisfaisant les critères $\mathrm{C} 1, \mathrm{C} 2$ et $\mathrm{C} 3$ : une définition précise et rigoureuse incluant les instances prototypiques de JE (autrement dit, une définition qui a une dimension descriptive) pourra éventuellement devoir accepter des jeux qui ne sont pas communément considérés comme des JE par la communauté (et acquérir une dimension normative qui vise à ajuster certains usages linguistiques). Par ailleurs, comme nous le verrons, il peut exister plusieurs définitions différentes qui remplissent les critères $\mathrm{C} 1$ et C2. Par conséquent, certains jeux qui ne sont pas des exemples prototypiques de JE pourront être considérés comme JE selon une définition, mais pas selon une autre. Enfin, puisque notre définition du JE repose sur ce qui est considéré comme exemple prototypique de JE aujourd'hui, elle pourra être amenée à évoluer en fonction des usages.

12 Notons également que nous définirons la notion de JE, mais pas celle de « bon » JE. La qualité (bonne ou mauvaise) d'un JE est en effet une caractéristique très complexe, notamment à cause de la subjectivité qu'elle implique, et son analyse excède le cadre de cet article.

Pour chaque terme relatif au JE dans notre taxonomie, nous fournirons une définition dite "aristotélicienne", une méthodologie utilisée notamment dans le cadre des ontologies fondées sur la «Basic Formal Ontology [ontologie formelle de base ] (Arp et al., 2015 ). Ce type de définition prend la forme suivante: si B est une sous-classe de A, alors une définition aristotélicienne définira $\mathrm{B}$ comme " un $\mathrm{A}$ qui $\mathrm{D}$ », où $\mathrm{D}$ est dit le "differentia (specifica)». Ce schéma de définition remonte à Aristote, qui définissait l'homme comme un animal rationnel. Nous accepterons un certain nombre de termes de haut niveau comme primitifs, c'est-à-dire des termes que nous ne définirons pas (il serait d'ailleurs impossible d'en fournir une définition aristotélicienne ici, puisque ceci requérait d'introduire une catégorie de niveau encore supérieur). Le terme de «jeu», par exemple, est classiquement difficile à caractériser (Wittgenstein, 1953), et nous ne chercherons pas à le définir. En revanche, nous chercherons à caractériser ce qui, parmi les jeux, particularise le JE ; autrement dit, à trouver le differentia qui distingue le JE des autres formes de jeu. 


\section{Quelques classifications et ontologies existantes}

14 Il existe un certain nombre de classifications du jeu introduisant des concepts utiles pour situer le JE au sein du paysage ludique. Ainsi, Gunn et al. (2009) distinguent les taxonomies «ludologiques", qui s'intéressent aux mécanismes et structures des jeux, des taxonomies "narratologiques ", qui s'intéressent à leur sujet et contenu. Nous visons ici à établir une taxonomie ludologique.

15 Réutilisant la terminologie de Caillois (1955) situant les jeux sur un axe aux deux pôles ludus (jeux suivant des règles et conventions) et paidia (jeux reposant sur l'improvisation et la liberté du joueur), nous pouvons globalement situer les JE plus proches du côté ludus que du côté paidia, puisqu'il s'agit majoritairement de relever des défis préétablis dont les solutions sont prédéterminées - même si certains JE, tel que Les Ferrailleurs de l'Espace (Immersia, Paris) ou Recherche sous Tension (L'Escapatoire, Paris) laissent une certaine place à l'improvisation théâtrale des joueurs.

16 Enfin, il existe certaines ontologies du jeu, telles que OntoJogo (Teixeira et al., 2020), le Game Ontology Project (GOP) (Zagal et al., 2007), qui vise à fournir un cadre pour la description et l'analyse des jeux, ou le Game Content Model, une ontologie du jeu sérieux (Tang \& Hanneghan, 2011). Nous établirons dans l'article certains parallèles entre des notions que nous introduirons et des classes de ces ontologies. À notre connaissance, cependant, aucune d'entre elles ne définit la classe de "défi » (et ses sous-classes que nous introduirons ici), qui sera une notion centrale pour définir le JE.

\section{Définition du jeu d'évasion}

Nous proposerons ici la définition générale du JE suivante :

Jeu d'évasion : Jeu dans lequel un ou plusieurs joueurs incarnent un ou plusieurs personnages dans un environnement, où la consultation d'informations externes au jeu n'est pas autorisée, et qui repose sur la découverte et la résolution de défis préétablis de natures différentes, afin d'accomplir un ou plusieurs objectifs.

Comme expliqué en introduction, cette définition a vocation à être large, et à englober aussi bien le JEGN que le JE en boîte, les livres-JE et le JE vidéo (qui seront présentés dans la prochaine partie). Nous en expliquerons tout d'abord les termes clés, puis discuterons de critères que nous n'avons pas retenus dans cette définition, en mentionnant des exemples prototypiques de JE pour motiver nos décisions.

Un JE est joué par " un ou plusieurs joueurs » : la plupart des JEGN ont vocation à être joués par une équipe de plusieurs joueurs, mais certains sont conçus pour être joués par un seul joueur, comme Le Dernier Otage (Spirit Escape, Paris). Par ailleurs, un grand nombre de JE vidéo sont conçus pour n'être joués que par un seul joueur à la fois.

La notion d'« objectif » introduite ici recoupe celle de « goal » du Game Ontology Project (Zagal et al., 2007). Comme mentionné ci-dessus, l'objectif d'un JE n'est pas toujours l'évasion. Il existe notamment des JE où les joueurs n'ont pas tous le même objectif : par exemple, dans Room 25 (Epic Escape Games, Seattle) l'un des joueurs est un traître, et son objectif diffère de celui du reste de l'équipe. D'autre part, comme la définition l'indique, il peut y avoir plusieurs objectifs dans un même JE. Par exemple, dans le Secret 
du Pirate (HintHunt, Paris) les objectifs annoncés au début de l'aventure sont: lever l'ancre du bateau, trouver le saphir du capitaine, et sortir du bateau. L'atteinte de tous les objectifs n'est d'ailleurs pas toujours nécessaire pour gagner un JE : parfois, il suffira pour cela de remplir uniquement certains objectifs. Enfin, certains objectifs pourront venir en remplacer d'autres en cours de partie.

21 Nous reviendrons sur la notion de "défi » dans la section 5, mais pour fixer l'intuition, précisons que les défis peuvent incorporer notamment: des énigmes (par exemple résoudre un sudoku ou une devinette); de l'observation, parfois sous forme de fouille (trouver un document dans un manteau); des manipulations (faire passer un anneau le long d'un fil de fer sans le toucher); de la communication (un joueur doit lire un message écrit sur un mur avec des symboles ésotériques à un coéquipier situé dans une autre pièce qui a sous les yeux une table de traduction entre ces symboles et l'alphabet); ou même, plus rarement, des défis physiques (se balancer avec une corde d'une plate-forme à une autre, ou traverser une pièce en évitant de déclencher un système d'alarme à base de lasers). Cette succession de défis va différencier un JE en boîte comme Unlock!, par exemple, d'un jeu de société comme Sherlock Holmes - Détective Conseil, dans lequel chaque partie consiste à faire la lumière sur une affaire particulière en suivant des pistes multiples de manière à glaner des indices et reconstituer une explication plausible, plutôt que par la résolution d'une succession de défis. Remarquons que la variété des défis que l'on peut trouver dans un même JE rend difficile leur classification dans un genre unique tel que défini par l'ontologie OntoJogo (« Puzzle », « Action », « Roleplaying », « Adventure »...) (Teixeira et al., 2020).

Notons que selon notre définition, pour qu'un jeu puisse être qualifié de JE, certains des défis qu'il propose doivent être "de natures différentes » : nous verrons plus loin une première classification des défis permettant de dissocier leur nature, et en quoi ce critère est utile pour exclure du champ de la définition certains jeux vidéo à énigmes ("puzzle games»). De même, il est important de préciser que les joueurs vont "découvrir " les défis dans un jeu d'évasion, et qu'ils ne les connaissent pas avant de commencer leur partie. Ceci différencie notamment un JE en boîte d'un jeu de société collaboratif comme Pandémie, dans lequel on apprend les règles avant d'y jouer.

La notion de "personnage » est à mettre en rapport avec la notion d'« agent». Sans entrer dans les détails de la philosophie de l'action, on définira un « agent » comme une entité capable d'action, et l'« environnement» d'un agent comme une entité dans laquelle un agent évolue. Nous définirons ici un "personnage » comme une simulation d'agent qui évolue dans un environnement, réel ou simulé (pour une analyse plus détaillée de la notion de "personnage ", et son rapport avec celle d' "avatar ", voir Bourassa \& Poissant, 2013). Ce "personnage » est un cas particulier de ce que le Game Ontology Project nomme le «locus of manipulation », qui permet au joueur d'interagir avec l'environnement du jeu.

Dans un JEV, le personnage est généralement une simulation électronique d'un agent (qui peut éventuellement être représentée de manière indirecte en vue à la première personne), et l'environnement est simulé électroniquement ; notamment, Crimson Room propose une simulation visuelle électronique d'une chambre rouge. Dans un livre-JE et un JE en boîte, le personnage est souvent représenté via des informations ou consignes présentes dans le JE (par exemple: "Vous incarnez un prisonnier»), et l'environnement est habituellement simulé par des représentations picturales et textuelles. Remarquons que plusieurs joueurs peuvent partager le même personnage, 
notamment dans certains JE en boîte ou livre-JE. Ainsi, dans Escape from the Asylum (Andersen, Peshkov \& Pluzhnikova, Lifestyle Boardgames Ltd, 2019) tous les joueurs incarnent ensemble le même personnage au cours de chaque partie. En revanche, dans un JEGN, le joueur incarne généralement physiquement son personnage, et l'environnement est simulé physiquement également. Dans certains JEGN, le type de personnage incarné par le joueur ne sera que superficiellement spécifié - et parfois, le personnage ne sera autre que le joueur qui aurait basculé dans une réalité parallèle ("Vous venez de vous faire enlever dans la rue, et vous devez maintenant vous évader en moins de 60 minutes. »).

Notons enfin le critère spécifiant que « la consultation d'informations externes au jeu n'est pas autorisée ". Ceci permet ainsi d'exclure du champ du jeu d'évasion les chasses au trésor à distance (par exemple Sur la Trace de la Chouette d'Or) qui requièrent du joueur qu'il consulte des sources extérieures (encyclopédies, cartes géographiques, etc.). Ce critère ne signifie pourtant pas qu'aucune connaissance générale n'est utile voire nécessaire - dans un JE : on peut par exemple trouver dans l'environnement d'un JEGN un ouvrage fournissant la connaissance générale qui est requise pour résoudre une énigme. On ne peut pas non plus dire qu'aucune connaissance préalable n'est nécessaire à la résolution d'un JE : dans beaucoup de JE, il est par exemple nécessaire de savoir lire ou compter. On peut d'ailleurs trouver occasionnellement dans certains JEGN des énigmes nécessitant une connaissance générale de base - par exemple, savoir que le soleil se lève à l'est. Ces JEGN sont généralement conçus de manière à éviter que les équipes ne restent bloquées, en ayant par exemple un maitre du jeu qui fournira l'information sous forme d'indice supplémentaire en cas de besoin.

Remarquons que de nombreux jeux de pistes (notamment des jeux de géocaching) ne proposent pas au joueur d'incarner un personnage, et se retrouvent donc de fait exclus de la notion de JE. En revanche, certains jeux de piste impliquent d'incarner des personnages et satisfont notre définition : on pensera par exemple à Opération Mindfall (Lock Academy, Paris et Onirium, Nantes), qui se déroule dans les rues de la ville.

\section{Les sous-types du jeu d'évasion}

Le JE ayant été défini dans toute sa généralité, nous pouvons passer à la proposition de définitions aristotéliciennes pour les sous-types de JE. Nous utiliserons la taxonomie du JE suivante (avec entre crochets leurs abréviations).

Jeu

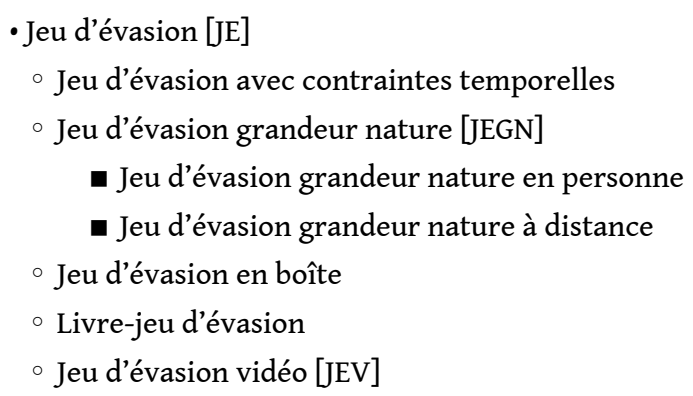

Notons que la première sous-catégorie immédiate de JE particularise le type de contrainte qui existe sur le jeu, alors que les quatre sous-catégories immédiates suivantes particularisent le type de simulation de l'environnement et de support du JE. 


\section{Jeux d'évasion avec contraintes temporelles} limité », qui concerne de fait beaucoup de JE. Pour les JEGN, cette durée est généralement de 60 minutes, quoiqu'un certain nombre d'entre eux proposent des durées inférieures (par exemple 45 minutes pour les scénarios d'Escape Manor à Ottawa ou Toronto) ou supérieures (par exemple 80 minutes dans Recherche sous Tension). Il peut par ailleurs y avoir des contraintes de temps supplémentaires spécifiques à certains défis (comme résoudre le dernier défi en moins de cinq minutes, indépendamment du temps qui restait à l'équipe jusque-là). Dans certains cas, le temps disponible va même varier de manière significative selon les actions des joueurs - comme dans L'Abri (Crack the Egg, Paris). Toutefois, il est discutable d'intégrer ce critère à la définition d'un JE. En effet, certains jeux qui sont fréquemment considérés comme des exemples prototypiques de JE n'ont pas de contraintes temporelles, notamment des jeux vidéo comme Crimson Room. Certains JE en boîte (comme Escape from the Asylum) prennent en compte le temps pris pour finir chaque partie comme un critère permettant d'évaluer la performance de l'équipe, mais n'imposent pas une limite de temps absolue. Même certains JEGN, comme The Prime Artifact (Artifact Escape Game, Clichy) n'intègrent pas de contraintes temporelles strictes.

C'est pourquoi nous n'avons pas mentionné de contrainte temporelle dans notre définition du JE ci-dessus, mais avons en revanche ajouté la sous-catégorie suivante dans notre taxonomie :

JE avec contraintes temporelles : JE dans lequel l'accomplissement des objectifs et/ou la résolution des défis est soumis à certaines contraintes temporelles.

Les raisons pour lesquelles la très grande majorité des JEGN intègrent des contraintes temporelles sont de trois sortes au moins. La première, c'est qu'une contrainte temporelle va influencer la prise de décision et les interactions entre joueurs. La deuxième raison est logistique et commerciale : un JEGN ne peut être joué (en général) que par une équipe à la fois, et l'investissement et les coûts (local, maitre du jeu, etc.) d'un JEGN ne pourront être rentabilisés qu'au-delà d'une certaine fréquence de groupes jouant l'aventure. Troisièmement, le fait de comptabiliser le temps permet d'ajouter un enjeu pour les équipes qui le souhaitent, à savoir être classée parmi les équipes qui ont résolu le JE en un minimum de temps. Par ailleurs, certains JEGN, comme ceux de la Lock Academy à Paris et Toulouse, vont adapter le niveau de difficulté des défis en fonction du temps qui reste: le paramètre temporel va donc parfois également influencer les défis eux-mêmes.

\section{Jeu d'évasion grandeur nature}

Le type de JE le plus connu est probablement ce qui est communément appelé «escape game» en France et «jeu d'évasion » au Québec, et que nous avons nommé ici «jeu d'évasion grandeur nature $» .^{5}$ 
Jeu d'évasion grandeur nature [JEGN] : Jeu d'évasion impliquant un environnement physique.

live escape room » ou «Remote escape game »), dans lequel les joueurs, qui ne se trouvent pas physiquement sur le site du JE, donnent des instructions (typiquement en utilisant un logiciel de visioconférence) à un employé de la structure organisatrice sur place, de manière à ce que celui-ci interagisse avec l'environnement comme le ferait un joueur dans un JEGN classique. Ces instructions peuvent être de fouiller une veste, d'ouvrir un coffre avec une clé trouvée auparavant, etc. Ce type de jeu a connu une expansion sans précédent en 2020 lors des confinements liés à la pandémie de COVID-19, de nombreux JEGN classiques se transformant pour l'occasion en JEGN à distance (comme Le Casse du Siècle, The Box, Metz). Mais ce type de JE à distance existait déjà auparavant (avec notamment l'entreprise YouEscape: Online Live Escape Room à Athènes). Nous définissons donc « JEGN à distance » (et inversement « JEGN en personne ») ainsi :

JEGN à distance : JEGN où les joueurs n'interagissent pas directement avec l'environnement physique du JE.

JEGN en personne : JEGN où les joueurs interagissent directement avec l'environnement physique du JE.

La correspondance entre le corps du joueur et le corps de son personnage dans un JEGN en personne implique ce que l'on pourrait appeler «l'inscription corporelle de la perception et de l'action » : la perception et les actions du joueur vont typiquement engager son corps comme cela serait le cas si cet environnement n'était pas simulé et qu'il était plongé dedans. ${ }^{6}$ Par exemple, pour regarder dans une certaine direction, il va devoir tourner la tête. De même, pour désamorcer une bombe fictive, le joueur va devoir couper les fils de cette bombe, comme il le ferait (ou du moins, comme il s'imagine qu'il le ferait) avec une bombe réelle. Au contraire, dans un jeu vidéo classique, la perception et l'action engageront le corps du joueur de manière différente, typiquement en pressant sur les boutons d'une manette, d'une souris et/ou d'un clavier. De même, dans un JE en boîte comme Unlock!, les éléments de l'environnement seront représentés sur des cartes étalées sur une table, dans une configuration spatiale qui ne reflète que très partiellement celle de l'environnement simulé - et seront donc perçus différemment d'un environnement équivalent qui ne serait pas simulé. L'action n'engagera pas non plus le corps des joueurs de la même manière que celui de leur personnage : pour que leur personnage ouvre un cadenas avec une clé, le joueur va devoir ajouter le numéro de la carte représentant la clé au numéro de la carte 
représentant le cadenas, et piocher la carte ayant le numéro de la somme, qui lui indiquera qu'il a réussi à ouvrir le cadenas. C'est une action très différente de celle qu'il faudrait réaliser dans un JEGN en personne, où le joueur prendrait une véritable clé pour la mettre dans un véritable cadenas. Autrement dit, l'engagement corporel du joueur est directement lié à la forme de la simulation d'environnement proposée et à celle de son personnage.

\section{Jeu d'évasion en boîte et livre-jeu d'évasion}

D'autres formes de JE sont les livres-jeux d'évasion et jeux d'évasion en boîte, que l'on peut définir ainsi :

Livre-jeu d'évasion : Jeu d'évasion où les défis sont présentés majoritairement dans un livre.

Jeu d'évasion en boîte : Jeu d'évasion où les défis sont présentés majoritairement sur des supports stockés dans une boîte.

La définition précise "majoritairement ", car certains défis peuvent se trouver sur un autre support, comme une application mobile (c'est le cas pour certains JE en boîte de la série Unlock!).

Remarquons que les catégories de JEGN et de JE en boîte ne sont pas disjointes : ainsi, l'Escape Box Cthulhu est composé d'une boîte contenant un ensemble de cartes qui doivent ensuite être mis en place dans une pièce. L'environnement est donc simulé de manière partiellement physique : les bureaux, chaises, canapé etc. de l'espace physique choisi représenteront effectivement des bureaux, chaises, canapé, etc. de l'espace simulé. Certains objets seront quant à eux représentés par des cartes du jeu. Une fois mis en place, le jeu pourrait être considéré comme relevant à la fois du JEGN et du JE en boîte.

\section{Jeu d'évasion vidéo}

Passons maintenant à la définition du jeu d'évasion vidéo.

Jeu d'évasion vidéo [JEV] : Jeu d'évasion impliquant un environnement simulé électroniquement.

Rappelons que d'après la définition générale, un JE « repose sur la découverte et la résolution de défis préétablis de natures différentes ». Des jeux vidéo en pointer-cliquer classiques comme The Secret of Monkey Island (où la simulation de l'environnement est électronique) ne remplissent cet aspect de la définition de JE que très partiellement. Un tel jeu contient certes des défis, comme découvrir des objets et comprendre comment les utiliser, et dans quel contexte (avec une logique parfois absurde). Cependant, une grande partie du jeu consiste à explorer un environnement étendu, à suivre une narration et à interagir avec des personnages virtuels par le biais de conversations prérédigées à choix multiples. En revanche, des jeux comme Crimson Room ou The Room sont plus strictement limités à la résolution de défis préétablis, avec des environnements qui ont été essentialisés au profit des éléments nécessaires à la résolution des défis. 
Certains jeux en pointer-cliquer antérieurs à Crimson Room remplissent d'ailleurs la définition de JE de ce point de vue - tel que Myst.

La définition du JE permet de distinguer les JEV de certains jeux vidéo à énigmes ( " puzzle games» - une famille à laquelle on peut également rattacher Myst). Des jeux comme Portal ou The Talos Principle proposent certes une succession de défis de réflexion, mais ceux-ci sont de nature similaire tout le long du jeu : leurs modalités de résolution sont globalement identiques, et la progression de niveau en niveau s'accompagne de l'ajout de règles supplémentaires et/ou d'une difficulté croissante. Au contraire, un JE repose sur la variété des défis rencontrés.

Remarquons que la classification que nous avons proposée ne prétend pas être exhaustive - il existe d'autres formes de JE (« escape kit » à imprimer chez soi, etc.). Mais cette taxonomie permet déjà de catégoriser une très grande majorité de ce que l'on appelle communément « jeu d'évasion ».

\section{Retour sur la définition du jeu d'évasion}

Le champ du JE et ses sous-types ayant été clarifiés, nous pouvons maintenant revenir sur quelques éléments de la définition. Notons que certains défis typiques du JE, comme un message codé à décrypter, peuvent se retrouver dans des jeux vidéo d'aventure, des livres dont vous êtes le héros ou des soirées enquête. Toutefois, l'essentiel de la résolution des objectifs de ces types de jeu ne passe pas par une succession de défis de ce type : dans un jeu vidéo d'aventure, un livre dont vous êtes le héros ou un jeu de rôle, l'enjeu principal est l'exploration d'un univers ; dans une soirée enquête, il s'agira avant tout de résoudre une enquête policière à travers l'accumulation de multiples preuves. La résolution de défis préétablis n'y trouvera qu'une place marginale au plus.

Soulignons également que certains jeux peuvent être des hybrides entre JE et d'autres types de jeux: par exemple, si on exclut les jeux de pistes du domaine du JE (nous reviendrons sur ce point dans la conclusion de l'article), La Disparition de Watson (Gamescape, Paris) est un tel hybride, composé en partie d'un jeu de piste, et en partie d'un JE authentique. D'autres jeux remplissent parfaitement la définition de JE, mais sont des hybrides entre plusieurs sous-types de JE. Ainsi, Les Ferrailleurs de l'Espace et L'Abri sont des JEGN qui reproduisent une partie de l'environnement sous forme de jeu vidéo (sur écran pour le premier, et en réalité virtuelle pour le second), et sont donc également en partie des JEV.

\section{Tâches et défis}

\section{Définitions}

Le JE et ses sous-types ayant été définis, nous pouvons maintenant caractériser plus finement les entités-clés qui ont été mentionnées dans les définitions antérieures. Voici la taxonomie de celles-ci, que nous détaillerons ci-dessous.

Tâche de jeu

- Défi

- Défi de réflexion [énigme] 


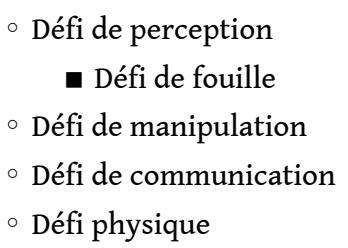
que personne n'y ait jamais joué : dans un tel cas, les tâches constituant le JE auraient tout de même existé. Pour rendre compte de ces tentatives de résolution, on pourrait introduire la notion d' » épreuve » comme un processus de tentative de réalisation d'un 
défi par un ou plusieurs joueurs (la distinction entre « défi » et " épreuve » étant du même ordre que la distinction entre " jeu comme artefact » et " jeu comme activité » proposée par Alvarez et al., 2017).

\section{Les sous-types de défi}

55 Nous pouvons maintenant passer à la classification des sous-types de défis fréquemment rencontrés dans les JE. Parmi les plus communs, on compte les défis de perception, de réflexion, de manipulation, de communication et les défis physiques. On peut définir les premiers ainsi :

Défi de perception : Défi dont la résolution est jugée impliquer une difficulté de perception significative.

$\mathrm{Au}$ sein des JE, un grand nombre de défis de perception implique la vue, mais certains impliquent l'ouïe (par exemple reconnaître une séquence de sons) ou le toucher (lire un message en braille). Notons également que tout défi impliquera une tâche de perception : pour résoudre une énigme, il faut d'abord la percevoir (typiquement en la lisant). C'est pourquoi la définition spécifie que cet effort doit être jugé significatif pour que le défi soit un défi de perception.

Un exemple particulièrement fréquent de défi de perception est le défi de fouille :

Défi de fouille : Défi de perception dont la résolution requiert de manipuler un ou plusieurs objets de la salle pour percevoir un ou plusieurs éléments cachés par ces objets.

Notons que tous les défis d'observation ne sont pas des défis de fouille. Ainsi, se mettre à genoux pour regarder sous un bureau est un défi d'observation, mais pas un défi de fouille; en revanche, ramasser une veste au sol et mettre la main dans une de ses poches pour en sortir une clé est un défi de fouille.

On pourra définir similairement "défi de réflexion» (synonyme de "énigme ») en remplaçant les termes «de perception » dans la définition des défis de perception par les termes "de réflexion" - et définir de même les défis de manipulation, de communication et physique.

Remarquons également que certains défis sont des instances de plusieurs de ces classes. Considérons un défi dans lequel un joueur doit manipuler en aveugle une canne à pêche pour ramasser un objet, pendant qu'un autre joueur, qui voit l'hameçon et l'objet, peut lui expliquer comment déplacer l'hameçon : il s'agit à la fois d'un défi de manipulation et de communication.

\section{Conclusion : Le jeu d'évasion et ses frontières}

61 Nous avons proposé une taxonomie du jeu d'évasion et des entités les plus centrales du domaine, enrichie de définitions aristotéliciennes. Nous avons vu, grâce à certains exemples prototypiques, que des dimensions fréquemment mentionnées comme constitutives du jeu d'évasion ne sont en fait pas essentielles, comme celles de collaboration et d'enfermement: les termes "escape " et «évasion» sont donc des 
reliquats historiques, dont la restriction sémantique n'a plus lieu d'être. D'après la définition que nous avons proposée, les JE incluent non seulement les JEGN (en personne et à distance), mais aussi les livres-JE, les JE en boîte et les JE vidéo, ainsi que certaines expériences immersives qui s'éloignent en partie des codes du jeu d'évasion classique (telles que Panik Room ou Prison Island).

Nous avons également défini la notion de défi et certains de ses sous-types. Comme expliqué précédemment, on peut trouver des défis dans de nombreux types de jeux qui ne satisfont cependant pas tous les critères de notre définition du JE : parmi les jeux comprenant des défis de réflexion sans être pour autant des JE, on compte par exemple une chasse au trésor comme Sur La Trace de La Chouette d'Or (qui autorise les sources d'information externes au jeu), des jeux vidéo à énigmes comme The Talos Principle ou Portal (qui reposent sur des défis de même natures), un jeu de société comme Pandémie (qui est un grand défi de réflexion connu lors de la lecture des règles, plutôt que découvert pendant le jeu), et des jeux de société comme Sherlock Holmes - Détective Conseil, ainsi que certains livres dont vous êtes le héros ou soirées enquêtes (qui peuvent contenir occasionnellement des défis de réflexion, tels que des messages à décoder, mais ne reposent pas principalement sur la résolution de tels défis).

Remarquons que l'exigence que les défis d'un JE soient «de natures différentes » pourrait être interprétée de plusieurs manières. Une condition suffisante pour que des défis soient de natures différentes serait qu'ils appartiennent à différentes catégories que nous avons identifiées ici telles que " défi de perception », « défi de réflexion », etc. Toutefois, on pourrait alternativement considérer que des défis de réflexion qui impliquent des mécanismes cognitifs de natures différentes (par exemple, des devinettes et des sudokus) sont de natures différentes.

Nous avons vu que certains jeux antérieurs à l'émergence contemporaine des JE, comme le jeu vidéo Myst, ou dans une moindre mesure d'autres jeux point-and-click, pourraient remplir la définition du JE proposée ici. La raison pour laquelle Crimson room, plutôt que Myst, est souvent reconnu comme l'un des premiers jeux d'évasion est à notre avis historique. Reprenant le concept de Crimson Room, les premières formes contemporaines du JE ont fixé cette idée que l'« enfermement » dans un espace clos est une condition nécessaire d'un JE (comme l'indique le nom "escape room »). Mais cette dimension d'enfermement n'est aujourd'hui plus constitutive des JE, et il est donc cohérent d'y inclure également des jeux vidéo précurseurs comme Myst. ${ }^{8}$

Remarquons qu'on pourrait vouloir réserver le terme de « jeu d'évasion » à ce que nous avons appelé un "JE avec contraintes temporelles", et rebaptiser ce que nous avons appelé «JE » en « jeu de simulation et de défis ». Ceci permettrait d'exclure du champ du JE des jeux vidéo à énigmes comme Portal ou The Talos Principle sans avoir besoin de préciser dans la définition que les défis doivent être "de natures différentes », et d'en exclure également des jeux comme Myst. Cependant, cela imposerait d'exclure également du champ du JE beaucoup de jeux vidéo communément considérés comme des JE, tels que Crimson Room (souvent considéré comme l'un des jeux fondateurs du genre), ou encore la série Cube Escape (Rusty Lake, depuis 2015). ${ }^{9}$ A l'inverse, on pourrait accepter une définition plus large du terme "jeu d'évasion", en supprimant la condition interdisant la consultation d'éléments externes au jeu. Les usages à cet égard sont en effet susceptibles d'évoluer dans le futur.

Par ailleurs, certains jeux de piste où les joueurs incarnent des personnages - tels qu'Opération Mindfall - satisfont notre définition du JE. On pourrait les exclure en 
ajoutant dans la définition du JE la précision que l'environnement dans lequel prend place un JE doit être « dédié à ce jeu " (au moins pendant le temps du jeu). Les deux définitions (inclusives et exclusives) nous semblent défendables, étant donné qu'il ne semble pas y avoir de consensus clair dans la communauté sur le fait que ces jeux tombent dans la catégorie du JE.

Notons que la mention qu'un JE doit « reposer » sur la découverte et la résolution de défis admet une certaine marge d'interprétation. Elle permet d'inclure les exemples prototypiques du JE considérés ici, et d'exclure des jeux où les défis occupent une place marginale, mais ne permet pas de déterminer ce qu'il en serait pour d'éventuels cas intermédiaires. Il s'agit ici d'une variante des célèbres paradoxes sorites (par exemple : à partir de combien de cheveux perdus une personne devient-elle chauve ?), et donc d'un problème plus général, qui excède la portée de cet article.

Notre analyse conceptuelle pourrait être complétée par l'étude des dimensions économiques et sociales qui ont mené à l'évolution des usages du terme «jeu d'évasion »- et notamment, comment celui-ci s'est libéré de la dimension d' "évasion » qui lui était initialement associée.

Enfin, on pourrait étendre cette taxonomie à d'autres notions clés du JE (dont certaines ont été mentionnées dans cet article), comme celles d'«objectif», d'« indice", d'« environnement de JE», de "personnage de JE», ou encore l'ensemble des agents intervenants dans un JE ( « joueur de JE », « équipe de joueurs de JE », « maître du jeu de JE », « agent d'accueil de JE », « acteur de JE », etc.). Les frontières de la taxonomie du JE ne demandent maintenant plus qu'à être repoussées.

\section{BIBLIOGRAPHIE}

ALVAREZ J., LIBESSART A. et HAUDEGOND S. (2017), « Le "jeu non sérieux", une activité improductive? ", Interfaces numériques, 3, pp. 391-408.

ARP R., SMITH B. et SPEAR A. D. (2015), Building Ontologies with Basic Formal Ontology, Cambridge, The MIT Press.

ARSENAULT D. et PICARD M. (2008), « Le jeu vidéo entre dépendance et plaisir immersif : les trois formes d'immersion vidéoludique ", Proceedings of HomoLudens : Le jeu vidéo : un phénomène social massivement pratiqué, pp. 1-16.

BARTON A. (2020) «Que sont les nouvelles expériences immersives? », Expérience Immersive, https://experienceimmersive.fr/escapedia/nouvelles-experiences-immersives

BOURASSA R. et POISSANT L. (2013), Avatars, personnages et acteurs virtuels, Sainte-Foy, PUQ.

BUCAILLE R. et LEVENQ F. (2018). En quoi l'escape game ou jeu d'évasion pédagogique est-il un outil favorisant l'engagement des élèves dans l'apprentissage ?, Communication au qolloque en sciences de l'éducation, humaines et sociales (CSEHS), Dijon.

https://cucdb.fr/wp-content/uploads/2018/12/En-quoi-1\%E2\%80\%99escape-game-est-il-un- 
outil-favorisant-l\%E2\%80\%99engagement-des-\%C3\%A91\%C3\%A8ves-dans-

l\%E2\%80\%99apprentissage-Rachel-Bucaille.pdf

CAILLOIS R. (1955), « The structure and classification of games », Diogenes, 3, pp. 62-75.

ERMI L. et MÄYRÄ F. (2007), « Fundamental components of the gameplay experience: Analysing immersion ", in S. De Castell \& J. Jenson (dir.), Worlds in play: International perspectives on digital games research, Berne, Peter Lang Publishing, pp. 37-53.

GUIGON G., HUMEAU J. et VERMEULEN M. (2017), « Escape Classroom : un escape game pour l'enseignement », 9e Colloque Questions de Pédagogie dans l'Enseignement Supérieur (QPES), Grenoble. https://hal.archives-ouvertes.fr/hal-01503763/document

GUNN E., CRAENEN B. et HART E. (2009), « A taxonomy of video games and AI », Conference Adaptive and Emergent Behaviour and Complex Systems, pp. 4-14.

MASOLO C., BORGO S., GANGEMI A., GUARINO N. et OLTRAMARI A. (2003), « The WonderWeb library of foundational ontologies and the DOLCE ontology. WonderWeb (EU IST project 2001-33052) deliverable D18 ", LOA-ISTC-CNR, http://www.loa.istc.cnr.it/wp-content/uploads/ 2020/03/D18inv.31-12-03.pdf

NICHOLSON S. (2015). Peeking behind the locked door: A survey of escape room facilities, https:// scottnicholson.com/pubs/erfacwhite.pdf

SOLINSKI B. (2017), « A la marge de la lecture et du ludique : les livres-jeux », Sciences du jeu, 7, https://journals.openedition.org/sdj/777.

SPIRA L. (2019), « “Escape Room” Enters-Merriam Webster Dictionary », Room Escape Artist, https://roomescapeartist.com/2019/09/25/escape-room-enters-merriam-websters-dictionary/

TANG S. et HANNEGHAN M. (2011), « Game content model: an ontology for documenting serious game design », in 2011 conference Developments in E-systems Engineering, Dubai, pp. 431-443.

https://ieeexplore.ieee.org/document/6149971

TEIXEIRA S., BOAS R. V., OLIVEIRA F., ARAÚJO C. et HENRIQUES P. R. (2020), « OntoJogo: An Ontology for Game Classification », 8th International Conference on Serious Games and Applications for Health (SeGAH), pp. 1-8, https://ieeexplore.ieee.org/document/9201876.

VARZI A. (2019), « Mereology », in E. N. Zalta (ed.). The Stanford Encyclopedia of Philosophy, Stanford, Stanford University, https://plato.stanford.edu/archives/spr2019/entries/mereology/

WITTGENSTEIN, L. (1953), Philosophical investigations, Oxford, Blackwell.

ZAGAL J. P., MATEAS M., FERNÁNDEZ-VARA,C., HOCHHALTER B. et LICHTI N. (2007), « Towards an ontological language for game analysis ", in S. De Castell \& J. Jenson (eds.), Worlds in play: International perspectives on digital games research, Berne, Peter Lang Publishing, pp. 21-36.

\section{NOTES}

1. Pour leurs commentaires éclairants, nous remercions trois évaluateurs anonymes ainsi que : Audrey Barton, Barbara Beranek, Alexandra Bercoff, Virginie Leurs, Arnaud Rosier, Jérémie Vidal et Mickaël Wajnberg.

2. Voir par exemple la rubrique "Immersion inclassable» sur le blogue Expérience immersive : https://experienceimmersive.fr/immersion-inclassable

3. https://www.the-escapers.com/ 


\section{4. https://topescaperoomsproject.com/}

5. On l'appelle en anglais "escape room", "real escape game", "live escape game ", ou même également "escape game ", par restriction sémantique. Remarquons que le terme "escape room » peut lui aussi prêter à confusion, beaucoup de JE se déroulant dans plusieurs salles.

6. Remarquons que cette inscription corporelle de l'action et de la perception renforce ce qu'on appelle l' «immersion», et plus précisément les formes qui ont été appelées «immersion sensorielle » et «immersion systémique » (Ermi \& Mäyrä, 2007; Arsenault \& Picard, 2008; Barton, 2020).

7. On pourrait par ailleurs voir un JE comme un tout constitué de parties que sont les tâches. En ontologie, cette relation de «tout à partie» est largement étudiée par ce qu'on appelle la «méréologie » (Varzi, 2019).

8. Qui implique d'ailleurs également, quoique de manière moins visible, un objectif d'évasion.

9. Certains jeux vidéo, comme Keep Talking and Nobody Explodes (Pestaluky, Kane \& Fetter, Steel Crate Games, 2015), dans lequel deux joueurs doivent communiquer et collaborer pour désamorcer une bombe en temps limité, resteraient cependant dans le champ du JE, même en adoptant cette définition plus restrictive.

\section{RÉSUMÉS}

Nous proposons une taxonomie du jeu d'évasion complétée de définitions de termes-clés du domaine. Nous définissons un jeu d'évasion comme un jeu dans lequel des joueurs incarnent des personnages dans un environnement, où la consultation d'éléments externes au jeu n'est pas autorisée, et qui repose sur la découverte et la résolution de défis préétablis de natures différentes, afin d'accomplir un ou plusieurs objectifs. Nous définissons plusieurs sous-types du jeu d'évasion (grandeur nature, jeu vidéo, livre-jeu et jeu en boîte), ainsi que la notion-clé de «défi » et certains de ses sous-types. Cette définition exclut du champ du jeu d'évasion des formes de jeux partiellement similaires comme les soirées enquêtes, les chasses au trésor à distance, ainsi que certains jeux de société collaboratifs et jeux vidéo de réflexion. Nous discutons de la pertinence d'imposer un critère de contrainte temporelle dans la définition, ainsi que de la possibilité d'inclure ou non dans le champ du jeu d'évasion certains jeux de pistes et jeux vidéo de type « point-and-click » antérieurs à l'essor contemporain des jeux d'évasion.

We propose a taxonomy of escape game completed with definitions of key terms in the domain. We define an escape game as a game in which players embody characters in an environment, where the consultation of elements external to the game is not allowed, that rests on discovering and completing predefined challenges of different natures, in order to accomplish one or several objectives. We define several subtypes of escape games (live game, video game, game book and game in a box), as well as the key notion of "challenge" and some of its subtypes. This definition excludes partially similar forms of games from the field of escape game, such as murder parties or armchair treasure hunts, as well as some collaborative board games and puzzle video games. We discuss whether or not to include in the field of escape games some scavenger hunts and point-and-click video games developed before the contemporary rise of escape games. 
INDEX

Keywords : taxonomy, definition, applied ontology, escape game, video game, simulation, action Mots-clés : taxonomie, définition, ontologie appliquée, jeu d'évasion, jeu vidéo, simulation, action

\section{AUTEURS}

\section{ADRIEN BARTON}

CNRS, Institut de recherche en informatique de Toulouse (IRIT)

\section{QUENTIN RANO}

Chercheur indépendant 